\title{
Encore un mal que le roman nous fait? Morale du dilemme, de Hugo à Dostö̈evski
}

La pensée de Victor Hugo est la pensée essentielle de tout l'art au XIX siècle, et cette pensée, Victor Hugo en tant qu'artiste en a peut-être été le premier annonciateur. C'est une pensée chrétienne et hautement morale ; sa formule est la "restauration" de l'homme perdu, injustement écrasé par le joug des circonstances, l'immobilisme des siècles et des préjugés sociaux. Cette pensée est la justification des humiliés et des parias de la société, repoussés par tous... Victor Hugo est probablement le principal annonciateur de cette idée de "restauration " dans la littérature de notre siècle. Pour le moins, c'est lui qui a exprimé cette idée avec une telle puissance dans l'art. ${ }^{1}$

La littérature de fiction a toujours représenté le monde des criminels avec sympathie, parfois avec complaisance. Elle a paré la pègre d'une auréole romantique, se laissant séduire par son clinquant de pacotille. Les artistes n'ont pas su discerner le véritable et répugnant visage de cet univers. C'est un péché pédagogique. [...] Par un caprice de l'histoire, les apôtres les plus éloquents de la conscience et de l'honneur, comme Victor Hugo, par exemple, ont tout fait pour porter aux nues l'univers des malfaiteurs. Hugo avait l'impression que le monde du crime était une couche de la société qui protestait vigoureusement, résolument et ouvertement, contre l'hypocrisie de l'ordre régnant. Mais Hugo ne s'est pas donné la peine d'examiner pourquoi cette communauté de voleurs combattait

1. Dostoïevski, Euvres littéraires complètes, t. XIII, Articles. Moscou-Leningrad, 1930, p. 326 (préface de Notre-Dame de Paris), cité par G. Fridlender dans son article intitulé «Les notes de Dostö̈evski sur Victor Hugo ", dans le Cahier de l'Herne Dostö̈evski dirigé par Jacques Catteau, l'Herne, p. 291. 
n'importe quel pouvoir en place. Bien des garçons ont cherché à rencontrer des "misérables" en chair et en os après avoir lu ses romans. Le surnom de Jean Valjean existe encore aujourd'hui chez les truands. ${ }^{2}$

Séparées par presque un siècle, ces deux citations préliminaires manifestent deux perceptions très contrastées de la réception morale des romans de Hugo. La première est de Dostoïevski : l'auteur de Crime et Châtiment inscrit explicitement son propre roman dans la filiation littéraire et morale des Misérables qu'il admire. La seconde citation est tirée d'un célèbre chapitre des Récits de la Kolyma de Chalamov, "À propos d'une faute commise par la littérature ", qui ouvre la série de fragments sur le monde de la pègre dans les camps du goulag. L'écrivain zek y développe un réquisitoire très dur contre la littérature, depuis Hugo jusqu'aux écrivains enrôlés par le régime soviétique pour célébrer les campagnes de rééducation des truands par le travail, en passant par Dostoïevski, Gorki, et la littérature russe des années vingt, "gagnée par la mode des malandrins " et la "poétisation effrénée des malfrats ": accusée de s'être laissée mystifier en " parant la pègre d'un masque romantique et en entretenant chez le public une idée complètement fausse de cet univers abject et répugnant qui n'a rien d'humain ", la littérature a contribué, accuse Chalamov, à accorder un répit à des milliers de professionnels du vol, et porte une responsabilité historique très grande dans la gangrène morale de l'univers des camps où la pègre règne en maître.

Les deux jugements ont en commun de désigner, à partir de la filiation hugolienne, la grille de lecture morale comme principale signification du roman de la fin du XIX ${ }^{\mathrm{e}}$ siècle. Pour Dostoïevski, le thème hugolien de la restauration de l'homme déchu a des vertus morales auxquelles lui-même fait intentionnellement allégeance en rédigeant Crime et Châtiment. Pour Chalamov, Hugo a donné à la tentation de la représentation romantique des malfrats des lettres de noblesse aux conséquences désastreuses : le mal que la filiation "hugolienne" nous a fait est impardonnable. Entre ces deux appréciations du legs hugolien, le lien est évident quoique paradoxal : l'accusation de Chalamov et la louange de Dostoïevski tiennent à un même motif. Ce que Chalamov reproche à Hugo, dans les années 1950, c'est ce que Dostö̈evski, presque un siècle plus tôt, admirait en lui et déclarait reprendre à son compte dans Crime et Châtiment : la pensée "chrétienne et hautement morale " de la " restauration de l'homme perdu ».

À première vue, pour un lecteur contemporain, l'accusation de Chalamov paraît injuste et abusive à l'égard de Hugo. On comprend bien de quoi il retourne, il y a en effet une représentation romantique du truand qui cultive la fascination et dissout toute perception de la culpa-

2. Varlam Chalamov, Récits de la Kolyma, Verdier, coll. «Slovo», p. 869 
bilité au profit d'une réhabilitation fondée sur l'héroïsme, la force d'âme, ou l'injustice sociale. La transformation du mythe de Caïn, passé de " coupable et réprouvé » à "victime et révolté » à l'époque romantique en donne la mesure. Mais Hugo n'est pas le seul ni le premier "coupable " de l'héroïsation des bagnards dans la littérature française, même si son Jean Valjean est assurément le plus innocent d'entre tous. En outre, Hugo ne verse pas dans un angélisme primaire : Jean Valjean est véritablement converti au bien, mais il y a, dans Les Misérables, des méchants incorrigibles, Chalamov l'oublie un peu vite: les "misérables " ne sont pas tous des malheureux victimes des conditions sociales, il y a aussi de mauvaises gens, des Thénardier, qu'aucun retournement de l'existence ne fait évoluer, et dont la méchanceté tenace et définitive pose à Hugo une énigme face à laquelle il avoue son impuissance.

Le jugement de Chalamov sur Hugo relève-t-il pour autant d'un contresens ? Il soulève un problème fondamental, qu'une relecture attentive des Misérables ne saurait résoudre : celui de la portée de l'exemplarité littéraire. Pour un Jean Valjean réellement bon, combien de faux Jean Valjean ? Ce que lui reproche Chalamov, ce n'est pas d'avoir représenté un bon bagnard, mais d'avoir représenté un bon bagnard au risque d'une exemplarité qui fait partie de son projet littéraire, et qui a réussi au-delà de ses intentions et de ses espérances initiales. Tout le roman des Misérables est en effet construit sur l'enjeu de l'exception morale : un forçat repenti et converti au bien est une exception, certes, mais cette exception suffit à militer en faveur de l'abolition de toute damnation éternelle. C'est le même raisonnement que pour la lutte contre la peine de mort dans laquelle Hugo s'est également illustré. Dans le pari de l'exemplarité littéraire, l'exception morale ne confirme pas la règle, elle l'infirme. Audelà même du seul personnage de Jean Valjean, tout le roman de Hugo tient sur une série de dérogations morales : Javert, le policier qui a voué sa vie à la défense de la Loi et étouffé en lui tout sentiment personnel, se découvre humain ; Sour Simplice, la religieuse qui n'avait jamais menti, pour qui "mentir, c'est l'absolu du mal", ment pour sauver Jean Valjean... Le chemin que se taille le roman de Hugo circule avec légèreté entre exception et exemplarité, non sans péril. On commence alors à cerner le signal d'alerte que Chalamov nous envoie à partir de l'exemple hugolien : n'ouvre-t-il pas une brèche dans laquelle toute une littérature, depuis, s'est engouffrée?

Dostoïevski est le premier à se glisser dans cette brèche, opérant à partir du modèle hugolien une subversion morale d'une grave portée. Les différents points de ressemblance entre Dostoïevski et Hugo - schéma général de restauration de l'homme perdu, dilemmes pris en charge par la délibération intérieure, théorie de l'exception et de la dérogation morale - sont en effet systématiquement subvertis par le traitement qu'en 
offre le roman dostoïevskien. C'est à lui que l'analyse comparatiste sera consacrée.

Selon Dostoïevski, le schéma moral de l'intrigue des Misérables serait "la grande idée de l'art du XIX siècle", une idée qu'il reprend à son propre compte. Tout un pan de la critique lui a emboîté le pas en donnant de Crime et Châtiment une lecture morale chrétienne: chute, souffrance, rédemption, ou mort et résurrection, autour du pivot de l'aveu de Raskolnikov. Raskolnikov, comme Jean Valjean, est un homme déchu qui connaît une révélation et, dans la souffrance, entreprend de revenir vers les hommes. Pourtant, cette analogie de structure entre Hugo et Dostoïevski, est en fait un parallélisme trompeur, qui ne résiste pas à la lecture des œuvres : le sujet de Crime et Châtiment n'est pas la restauration d'un homme tombé, mais celui d'" une nouvelle histoire", qui commence dans les toutes dernières lignes du roman, dans le dernier paragraphe de l'épilogue, quand Raskolnikov, nous dit le narrateur, commence enfin à revivre grâce à l'amour de Sonia ${ }^{3}$. Et l'analogie en trompel'œil ne tient pas qu'à un déséquilibre de construction : la conversion de Raskolnikov n'est pas seulement tardive, ou retardée, elle est activement refusée durant l'essentiel du roman. Nul remords ne trouble Raskolnikov: Crime et Châtiment est bien davantage le récit d'une résistance à la régénération.

Première différence donc par rapport au modèle hugolien: c'est moins la société qui résiste à la restauration, que le criminel lui-même qui s'y refuse. Pour lui en effet, et jusqu'à la fin de l'épilogue, le meurtre commis n'est pas une faute morale (vina), mais un ratage (promakh). Jusque dans les dernières pages du roman, l'idée que Raskolnikov se fait de son crime demeure en deçà de toute prise de conscience morale. Ou, pour emprunter à Paul Ricoeur les catégories descriptives de ce qu’il nomme stades prééthiques de la faute, Raskolnikov en reste au premier degré, celui de la sensation de la souillure ${ }^{4}$ : pendant et après le double meurtre, l'obsession de l'impureté du sang, qui infecte et salit, est vécue dans le dégoût de la tache, la crainte de la contamination, la terreur de l'infection par contact. Et la vengeance de l'interdit violé retombe sur l'homme en mal de souffrance. Le deuxième stade de la conscience prééthique de la faute selon Ricoeur, celui du péché, n’apparaît que dans la bouche de Sonia, qui met sur le symptôme de séparation de Raskolnikov

3. «Mais là commence une nouvelle histoire, l'histoire du renouvellement progressif d'un homme, l'histoire de sa progressive régénérescence [...] Cela pourrait faire le thème d'un nouveau récit, mais notre récit présent touche à sa fin » Dostoïevski, Crime et Châtiment, Actes Sud, coll. «Babel », trad. Markowicz, t. 2, p. 475. Toutes les citations de Crime et Châtiment données plus loin renvoient à cette édition en deux tomes $(C C 1$ et $C C 2)$

4. Paul Ricoeur, Finitude et culpabilité, Aubier, livre II « La Symbolique du Mal », première partie «Les symboles primaires : souillure, péché, culpabilité ». 
les mots de déviation, de perte d'un lien, de révolte, ou de déréliction... Quant au troisième stade, celui de la culpabilité, le moment subjectif de la faute, il n'apparaît pas dans le roman. Il n'y a pas trace de repentir chez Raskolnikov. Il n'y a aucune référence à un contenu éthique du meurtre, la vieille usurière n'est jamais réhabilitée comme être humain. Le héros de Dostoïevski garde la nuque raide. La "grande idée de l'art au XIX siècle ", les vertus morales du schéma de chute/conversion/rédemption emprunté à Hugo, sont un trompe-l'œil. Pire encore : non seulement Raskolnikov ne reconnaît pas sa faute, mais son crime est même, dans une large mesure, justifié et légitimé à ses propres yeux - et à ceux du lecteur tout au long du roman. "Une mort contre cent vies"? Quand on referme le roman, et que, confronté à la difficulté de donner les raisons de l'acte de Raskolnikov, on cherche, sinon à expliquer, du moins à décrire par quel cheminement intérieur il en est arrivé à commettre son meurtre, c'est cette formule qui s'impose tout naturellement. Ce slogan, qui n'est pourtant nullement la raison du meurtre de Raskolnikov, parvient à s'imposer dans la mémoire du lecteur comme le dilemme structurant toute l'intrigue.

C'est que la déconstruction du parcours éthique, chez Dostoïevski, tient largement au traitement de cet autre instrument d'élaboration de la morale hugolienne qu'est le dilemme. Une poétique du dilemme se laisse en effet repérer chez les deux romanciers : l'intrigue se structure autour de dilemmes moraux, auxquels les personnages se confrontent au travers de longs dispositifs de délibération intérieure. Ces dilemmes constituent, pour tout lecteur de Hugo, des points d'accroche de la mémoire du roman : "Tempête sous un crâne ", "Javert déraillé "... toute une nuit d'angoisse et de perplexité pour Jean Valjean alias Monsieur Madeleine qui hésite entre partir se dénoncer au procès d'Arras, au risque d'abandonner Cosette à son triste sort, ou tenir la promesse faite à Fantine d'aller sauver sa fille, en laissant un innocent subir sa peine ; de longues minutes d'angoisse pour Javert qui, devant la maison du grand-père de Marius, va finalement décider de laisser la vie au forçat en rupture de ban qui lui a précédemment fait grâce de la sienne... Mais on se souvient aussi de l'épreuve imposée par la narration à Sœur Simplice, cette religieuse qui n'avait jamais menti, pour qui Satan est l'autre nom du mensonge, et qui ment deux fois coup sur coup pour sauver Jean Valjean des griffes de Javert. Ou encore, sur la barricade, du discours terrible de Combeferre sur le devoir envers les femmes, les enfants, les mères, quand il s'agit de désigner quatre hommes à sauver malgré eux de la mort certaine qui attend tous les insurgés... Qui doit vivre quand tous ne peuvent pas vivre? Chez Hugo, s'ils désignent de très modernes contradictions des principes, les dilemmes sont intégrés à la narration sous forme de délibérations intérieures et font progresser l'intrigue, suscitant à 
chaque fois des prises de décision qui participent à l'élaboration exigeante d'une morale en actes. Or, si le dilemme est, chez Hugo, un instrument de clarification morale, il devient chez Dostoïevski un instrument de brouillage et de mauvaise foi.

Raskolnikov, comme Jean Valjean et la plupart des personnages hugoliens, est un champion de la délibération intérieure. Mais son usage du dilemme est manipulateur et biaisé. La fameuse scène de l'aveu à Sonia en fournit un exemple significatif. Venu retrouver Sonia chez elle, après la scène terrible du scandale causé par Loujine lors du repas de funérailles de son père, Raskolnikov commence par torturer la jeune femme : «Et moi, justement, ce qu'il m'intéressait de savoir, c'était comment, maintenant, vous auriez résolu une "question", comme dit Lébéziatnikov. [...] Imaginez, Sonia, que vous connaissiez à l'avance toutes les intentions de Loujine, que vous sachiez (à coup sûr) qu'avec elles, ce serait la mort certaine pour Katérina Ivanovna, et pour les enfants ; et pour vous aussi, en plus [...] Polétchka pareil... le même chemin pour elle. Bon, et donc : et si, soudain, tout ça, on vous le donnait à juger : est-ce qu'il doit vivre, lui, sur terre, ou non, c'est-à-dire Loujine, vivre et faire ses saloperies, et que ce soit à Katérina Ivanovna de mourir? Qu'est-ce que vous auriez décidé : à qui de mourir ? Je vous pose cette question. " ${ }^{5}$ Venu pour avouer son crime, Raskolnikov substitue à l'aveu un dilemme qu'il pose à Sonia. Mais c'est une manipulation. En rhétorique, un dilemme est une alternative de choix négatifs, définis comme incompatibles, fondés sur le principe du tiers exclu, et qui conduit à opter, entre deux maux, pour le moindre. On se souvient de l'alternative que se pose Rodrigue, dans le Cid, entre l'exigence de l'amour et celle de l'honneur. Au fil des stances, Rodrigue s'aperçoit que laisser l'affront impuni, c'est non seulement trahir l'honneur, mais aussi perdre l'estime, donc l'amour de Chimène, et il choisit alors de venger son père en tuant celui de Chimène. Une même composante idéologique, un même code de l'honneur, régit en fait les deux branches de l'alternative. Raskolnikov, lui, dit à Sonia : à vous de décider, lequel doit vivre - si Loujine vit, Katérina et les enfants meurent, si Loujine meurt, Katérina et les enfants vivent. Mais ce dilemme vise à faire endosser à Sonia la justification de son propre crime. Soit elle se décide, et elle légitime le principe "une mort contre cent vies ": Raskolnikov, venu pour avouer son crime, peut alors recevoir l'absolution pour son acte courageux et légitime. Soit elle décide de ne rien décider, de ne pas agir, et alors elle consent à la mort de Katérina et des enfants. Contre ce dilemme destiné à l'enfermer dans un piège, Sonia se défend sur le plan des prémisses qu'elle conteste : cela ne peut dépendre de sa volonté - qui l'a désignée pour juger qui doit

5. CC2, p. 231-232. 
vivre? En contestant la composante idéologique sous-jacente de ce dilemme, Sonia lui ôte toute pertinence et en dénonce l'invalidité idéologique et psychologique. Elle dévoile ainsi d'un seul coup les valeurs sur lesquels se fondait Raskolnikov et sa mauvaise foi.

Or, cette rhétorique du dilemme manipulateur, Raskolnikov l'utilise aussi avec lui-même, dans le secret de ses monologues, dans l'intimité de son for intérieur. Si Raskolnikov se fait un problème de tout et n'importe quoi, son usage du délibératif intériorisé contient une grande part d'argumentation sophistique, de plaidoyer en faveur d'une ligne argumentative décidée à l'avance, à laquelle résiste encore une part de son être. De ce point de vue, les délibérations intérieures de Raskolnikov, mises en scène par Dostoïevski, sont une illustration parfaite de la thèse du linguiste Perelman arguant que tout est argumentation : bien loin que le secret de l'intimité soit un garant de sincérité, la délibération intériorisée doit être tenue pour une espèce particulière d'argumentation ${ }^{6}$. Un exemple crucial peut être trouvé dans les pages qui précèdent la décision du meurtre ${ }^{7}$. Raskolnikov reçoit une lettre de sa mère qui lui annonce le mariage imminent de Dounia avec le riche Loujine, et Raskolnikov comprend que sa sœur se sacrifie pour résoudre les difficultés financières de la famille et assurer son avenir à lui. Sa décision est aussitôt prise, ce sera le point fixe de toute sa délibération ultérieure: "ce mariage n'aura pas lieu, moi vivant ". Pour exciter sa colère contre le sacrifice que sa sœur s'apprête à faire, Raskolnikov se lance dans une analyse sémantique obsessionnelle de la lettre. Le narrateur a beau jeu de remarquer, comme incidemment, qu'il fait monter sa rage jusqu'au point où il serait capable de tuer Loujine s'il l'avait devant lui ${ }^{8}$. Le thème du meurtre, apparu là comme en passant, est prolongé par un autre indice, la référence à la vieille usurière, qui fait le lien entre la pénurie financière de sa famille, et la richesse avare de sa future victime: "deux petits billets, comme dit l'autre, la vieille, là... "9 Puis Raskolnikov démonte le mécanisme autosophistique du sacrifice de Dounia : il décrit alors très précisément le processus de pensée par lequel lui-même va en arriver, quelques pages plus loin, à justifier le crime qu'il prémédite, et qui consiste à se persuader que, pour sauver sa famille de la misère, pour accomplir de bonnes actions, la fin peut justifier les moyens. Les fragments de dialogue fictif dans lesquels le jeune homme, excédé, s'adresse à des interlocuteurs absents, rendent plus claire encore la valeur argumentative de ce monologue, qui a alors un seul objectif : lever toutes les résistances de sa conscience à l'idée du crime qui

6. Voir Chaim Perelman, L'Empire rhétorique. Rhétorique et argumentation, Vrin, coll. «Librairie philosophique »

7. $C C 1,1^{\text {re }}$ partie chap. 4 , p. 85 et suiv.

8. $C C 1$, p. 79.

9. CC1, p. 80 . 
germe en lui. Cette argumentation de mauvaise foi se radicalise peu à peu, en effet, jusqu'à se formuler sous l'apparence d'une alternative à tiers exclu, d'un dilemme de mauvaise foi. "Il était clair que, maintenant, le temps n'était plus à l'angoisse, à la souffrance passive, aux pures réflexions sur le fait que ces questions fussent insolubles, il fallait obligatoirement faire quelque chose et, ce, là, tout de suite, le plus vite possible. Il devait absolument se décider à quelque chose, même à n'importe quoi, ou... Ou, refuser la vie complètement ! s'écria-t-il soudain dans un état second [...] étouffer tout en soi, en renonçant à tout droit d'agir, de vivre et d'aimer! ${ }^{10} \mathrm{La}$ radicalité pathétique de ses formules à lui entre alors en résonance avec l'impasse existentielle formulée par Marmeladov devant lui la veille: "Est-ce que vous comprenez, est-ce que vous comprenez, mon bon monsieur, ce que ça veut dire, quand il n'y a plus nulle part où aller?" C'est alors, nous dit le narrateur, qu'une pensée "qu'il attendait déjà » le fait tressaillir. Pour que la décision du crime soit adoptée à l'unanimité par toutes les composantes de la conscience de Raskolnikov, il ne reste plus qu'à identifier ce " quelque chose à faire " avec l'idée du crime, ce qu'apparemment Raskolnikov répugne encore à faire. Quelques pages encore suffiront pour permettre à la chose de faire son chemin dans l'esprit de Raskolnikov. La rencontre de la jeune fille ivre, dont un homme mûr tente d'abuser, attise encore sa rage contre la misère qui menace sa sœur Dounia. Reste à éliminer comme inefficaces et dérisoires toutes les autres possibilités d'action (les leçons particulières fournies par Razoumikhine), puis "la chose " devient "ça ", et on apprend qu'elle mûrissait depuis un mois déjà. Le cauchemar de la jument massacrée atteste de la résistance de son inconscient à la résolution du meurtre : c'est le dernier combat de la conscience de Raskolnikov, mais c'est encore une argumentation: "Non, je ne supporterai pas, je ne supporterai pas! " ${ }^{11}$, proclame-t-il, mais c'est déjà une manière de se donner du courage, si l'on sait reconnaître, en dessous de la dimension assertive, la dimension argumentative de tout discours : "si, tu le feras!». Du reste, le lecteur attentif avait, dès les premières pages du roman, été averti par le narrateur de la mauvaise foi de Raskolnikov quand il se parle à luimême $^{12}$. Ne manque plus alors qu'un coup de pouce du destin, la conversation surprise entre Elizabeth et les marchands, conversation qui lui donne le feu vert pour le jour suivant. Le lendemain, tout est décidé, sa "casuistique " est "affilée comme un rasoir", il s'était persuadé que "ce projet qu'il avait réfléchi, ce n'est pas "un crime" " ${ }^{13}$.

10. $C C 1$, p. 87.

11. $C C 1$, p. 112.

12. «Malgré tous ses monologues pour se moquer de sa faiblesse et de son indécision », CC1, p. 14.

13. $C C 1$, p. 131-132. 
Comment en est-il arrivé là ? Ne nous manque-t-il pas un fragment de cette délibération intérieure, la partie casuistique justement de cette morale du crime? Un effet de composition a suppléé à la délibération manquante : le narrateur a en effet intercalé ici, dans la chronique de la dernière journée avant le meurtre, un épisode en flash back, le souvenir de la conversation entendue six semaines plus tôt dans une taverne, entre un étudiant et un officier, sur le droit de tuer "un pou ", " un cancrelat ", " pour une seule vie - des milliers de vies sauvées de la pourriture et de la décomposition ", " une mort, et cent vies en retour - mais c'est de l'arithmétique!" ${ }^{14}$ Tout se passe comme si, in extremis, le narrateur éprouvait le besoin de placer cette théorie justificatrice juste avant le déroulement du meurtre. Comme pour convaincre le lecteur? L'effet de composition donne en tout cas l'impression que c'est ce que Raskolnikov a en tête au moment où il s'apprête à tuer, alors que ces idées, proches des siennes mais formulées par autrui, sont antérieures de six semaines. Étonnant effet de brouillage, par lequel le narrateur se rend complice de l'argumentation criminelle de Raskolnikov... comme si son but était de rendre le lecteur prêt lui aussi.

Car le dilemme discuté par l'étudiant et l'officier dans la taverne, six semaines plus tôt, n'est pas la raison du crime de Raskolnikov, mais un cas d'école théorique abstrait, sur lequel l'image de la vieille usurière s'est greffée à titre d'illustration. La composition du chapitre attribue ce dilemme à Raskolnikov juste avant le meurtre, comme pour lui servir de caution. Et dans toute la suite du roman, ce dilemme central va, dès lors, planer au-dessus du double meurtre, avec l'aide de l'inspecteur Porphiri Petrovitch qui reprend un débat similaire avec Raskolnikov en résumant devant lui la thèse d'un article signé par lui six mois auparavant. L'effet de montage subvertit l'usage hugolien du dilemme comme outil d'une prise de décision, alors que - la scène de l'aveu à Sonia en témoigne - ce n'est pas la cause déclenchante de la décision du meurtre. Pire encore : sortie de son contexte, la formule " une mort, et cent vies en retour ", qui sert ici à couvrir un crime crapuleux, peut faire signe vers tout un contexte philosophique et moral dont Hugo discute la pertinence en contexte révolutionnaire, dans l'épisode de la barricade. La révolution a-t-elle le droit de verser le sang ? Enjolras exécutant l'assassin dissimulé dans les rangs des insurgés assume une souillure qu'il déteste. Qui doit vivre quand tous ne peuvent pas vivre? Qui doit se sacrifier quand tous n'ont pas besoin de se sacrifier ? C'était, on s'en souvient, la question posée par la possibilité de fuir la barricade offerte à cinq insurgés par les cinq uniformes de gardes nationaux susceptibles de servir de déguisement.

14. $C C 1$, p. 122 
Il y a là, dans l'exploitation de la filiation hugolienne et l'utilisation manipulatrice du dilemme, un ennoblissement du crime de Raskolnikov, matière à malentendu et à fascination. Combien de lecteurs de Crime et Châtiment y ont cédé ? En ce XX $\mathrm{XX}^{\mathrm{e}}$ siècle du communisme et des camps, la séduction de la morale du dilemme de Raskolnikov a pu facilement entrer en résonance avec les lignes de force idéologiques et politiques du totalitarisme soviétique. Sillage que le metteur en scène Lioubimov a désigné aux regards dans son adaptation théâtrale du roman sur la scène de la Taganka : à la fin de la pièce, le rideau se refermait sur les deux cadavres, tandis qu'un extrait de devoir d'écolier était lu à haute et forte voix par l'acteur qui jouait le rôle principal : "Raskolnikov a eu raison de tuer la vieille. Dommage qu'il ait échoué. " ${ }^{15}$

Chalamov a-t-il raison de dénoncer « une faute grave commise par la littérature" ? D'accuser Hugo d'avoir ouvert une brèche dans laquelle l'inhumanité a pu trop facilement trouver un sanctuaire idéologique? Avec Les Misérables, Hugo, en son temps, déclarait l'urgence de rouvrir un dossier et de réparer une injustice brûlante, celle de la damnation sociale éternelle des fauteurs, qui empêche tout espoir de rédemption morale. Jean Valjean était l'exception qui devait convaincre tout le monde de l'excessive dureté de la règle. Dostoïevski, en conscience, lui emboîte le pas : mais l'exception, de gage moral chez Hugo, devient chez lui, dans la bouche de Raskolnikov, ou plutôt, de l'inspecteur Porphiri Petrovitch qui en fait le compte rendu, l'objet d'une théorie périlleuse et

15. Voir l'article de Jean-Pierre Morel et Agnès Edel-Roy, " "Crime et Châtiment": du roman à la pièce » commentant cette adaptation théâtrale, dans Lioubimov : la Taganka, «Les Voies de la Création théâtrale n 20 », textes réunis et présentés par B. Picon-Vallin, CNRS, 1997, p. 255-263. Jean-Pierre Morel commente ainsi : «On ne sait si le devoir cité représente une opinion moyenne ou s'il est tenu pour exceptionnel, en tout cas la pièce se réfère in extremis au fait que des lycéens ont pu, à leur façon, non seulement absoudre Raskolnikov, mais comprendre et approuver son crime. [...] Le problème de la copie citée, c'est qu'elle en reste à l'absence de repentir et ne regrette que l'échec du meurtrier: on dirait que le début de la transformation de Raskolnikov, son regain d'intérêt final pour l'Évangile, n'ont jamais empêché une partie des lecteurs d'être plus ou moins clairement, sinon de connivence avec lui, du moins assez compréhensifs à l'égard du crime qu'il a commis; et c'est sur ce point que l'adaptation, en donnant la parole à un devoir scolaire, veut finalement insister. Elle ne se conçoit donc pas comme une transposition qui viendrait s'ajouter à d'autres, elle pose le problème de la réception de Crime et Châtiment à une période donnée: la période soviétique. Réception à laquelle l'allusion à l'école prête un caractère étendu, presque officiel. [...] Rejetant l'issue religieuse suggérée à la fin de l'épilogue (comme s'il s'agissait d'une échappatoire), Lioubimov, lui, n'en propose pas d'autre : c'est parce qu'il veut éclairer les éléments du texte qui ont pu susciter des sympathies inquiétantes dans une partie du public du $\mathrm{XX}^{\mathrm{e}}$ siècle qu'il insiste si délibérément sur le caractère inexpiable du double assassinat. Sans doute, officiellement, personne n'a jamais approuvé le meurtre de l'usurière et de sa sœur ni donné en exemple l'auteur du crime. Mais l'attitude compréhensive d'une partie des lecteurs du roman, voire d'un public plus large qui ne connaissait que les grandes lignes du récit, a pu se fonder sur le fait qu'il s'agissait d'un crime commis par un intellectuel, d'une action conçue par un esprit éduqué et cultivé, le tout au nom d'une idée qui, au départ, prenait en considération les rapports sociaux et l'inégalité des conditions. C'est cette lecture du roman, selon laquelle le crime idéologique paraît plus justifié, ou plus explicable, qu'un simple assassinat crapuleux, que l'adaptation de Lioubimov met à la fois en lumière et en cause. » 
excessivement séduisante, celle du droit de tuer. Il y a, dès le début, auprès de Raskolnikov, une Sonia pour avoir pitié de lui et voir sa souffrance derrière le mal commis par lui. Mais, parallèlement, il y a aussi un Porphiri Petrovitch, sorte de double du lecteur moderne, intelligent et intègre, mais fasciné par la complexité morale du criminel, prêt à discuter de ses idées... pour tenter de le sauver. Porphiri, c'est le risque hugolien porté à incandescence : ne se rend-il pas, in fine, complice du criminel ? Pour lui laisser une chance de rédemption, pour essayer de déclencher en lui cette restauration en laquelle il croit, pour espérer faire de lui un autre Jean Valjean, il lui propose un pacte qu'il respectera fidèlement, renonçant à l'inculper au terme de son enquête et lui laissant le mérite de l'aveu spontané qui lui procurera une diminution de peine. Pari hugolien, pari risqué, pari réussi, mais de justesse. La conversion au bien de Raskolnikov a-t-elle lieu ? À peine. Elle figure in extremis dans la fiction, à la marge de l'histoire du roman, dans les toutes dernières lignes. Elle aurait aussi bien pu ne pas avoir lieu.

À la Taganka, Lioubimov montrait sur la scène ce que Chalamov dénonçait avec d'autres mots : il y a, sinon chez les écrivains eux-mêmes, du moins dans la réception que leurs œuvres suscitent, une tentation de la fascination pour le mal et ses agents. Si, avec Hugo, le schéma chrétien ouvre, en littérature, la voie d'une naïveté peut-être coupable de laisser le lecteur fragilisé et mal armé pour prendre la mesure du mal qui menace son humanité même, le roman dostö̈evskien, tout en revendiquant sa filiation, pratique un brouillage moral plus dangereux encore. Le mal que le roman nous fait est une très vieille histoire, et très moderne.

(Université Paris X Nanterre) 